\title{
Mollugo cerviana (L.) Ser. en la Comunidad de Madrid
}

\author{
Leopoldo Medinaํㄹ \& Luis María Ferrero² \\ ${ }^{1}$ Real Jardín Botánico, CSIC, Plaza de Murillo 2, 28014 Madrid. \\ 2.E.S. San Agustín del Guadalix, Avda. Félix Rodríguez de la Fuente sn, 28750, San Agustín del Guadalix.
}

\section{Correspondencia}

L. Medina

e-mail: medina@rjb.csic.es.

Recibido: 19 noviembre 2017

Aceptado: 24 noviembre 2017

Publicado on-line: diciembre 2017
Mollugo cerviana (L.) Ser. in Madrid region (Spain)

Palabras clave: Molluginaceae, Mollugo cerviana, Madrid, España

Key words: Molluginaceae, Mollugo cerviana, Madrid, España
Mollugo cerviana (L.) Ser.

ESPAÑA, Madrid: San Agustín del Guadalix, Las Hazas, margen izdo. del río Guadalix, 30TVL4803, 624 m, en rastrojos, con Polycnemum arvense, 26-VII-2017, L.M. Ferrero s.n. (MA919263); ibídem, área recreativa de Los Patos, 30TVL4804, $630 \mathrm{~m}$, arenas, 6-VIII-2016, L. Medina LM9388 (MA-919264).

Mollugo cerviana (L.) Ser. es un taxon de amplia distribución, nativo en Eurasia, África y Oceanía (Gonçalves, 1990), e introducido en Estados Unidos (USDA-NRCS, 2017) y norte de Méjico (Vincent, 2003). En la Península Ibérica se encuentra disperso por la zona centro, al norte y al sur del Sistema Central (ANTHOS, 2017), con localidades muy dudosas en Asturias (Laínz, 1981) y Almería (Gandoger, 1896).

La primera cita a esta planta en la provincia de Madrid la hace Minuart (1739) siguiendo la nomenclatura de Tournefort: "Prato $S$. Isidori prope Amnem de Manzanares extra Mattitum, \& in.Praltis Saltûs de Luzon...", esta última localidad al sur de la ciudad de Madrid (Medina et al., 2015). Gómez Ortega (1772: 9) la menciona, como Pharnaceum cerviana L., cultivada en el Jardín Botánico, procedente de Vicálvaro. Su cultivo se mantiene en esta institución durante bastante tiempo, como recogen los diversos elencos que se publican posteriormente (Gómez Ortega, 1796: 23; Cavanilles, 1803a: 27; Lagasca, 1816: 12). Cavanilles (1803b: 390) ya había comentado su carácter espontáneo en el Jardín Botánico y su presencia frecuente en los alrededores de Madrid. En el siglo XIX las citas originales de esta especie en Madrid son escasas; Reuter (1843: 27) la indica de la planicie castellana y comenta su disyunción con las poblaciones de Crimea y el Cáucaso, pero los autores posteriores (Cutanda \& Amo, 1848; Colmeiro, 1849; Cutanda, 1861; Willkomm \& Lange, 1874-1880; Colmeiro, 1886: 421) apenas recogen las citas anteriores sin aportar novedades. La referencia de Löfling (1758: 27; ver Asso, 1801: 166-167) que recogen Willkomm \& Lange (18741880), y que repite Colmeiro (1886) es en el original un comentario sobre el nombre de Minuart y su pertinencia en el sistema lineano, sin una referencia geográfica clara a la planta en Madrid. De igual forma, la cita que recogen Willkomm \& Lange (1874-1880), y Colmeiro (1886), de Aranjuez y que tiene como referencia a Boutelou, quizá se refiera a las recolecciones de Claudio Boutelou, del que se conserva en el Herbario SEV como Phamaceum cerviana un material numerado como HHUS3985 (B-030) y con la indicación manuscrita de este botánico "Aranjuez, entre el canal y el río cerca del puente del ?" (Salgueiro González, 1998: 158). No hemos encontrado nuevas referencias de nuestro territorio hasta bien entrado el siglo $\mathrm{XX}$, en el que Pérez (1930) la indica de La Herrería, en las cercanías de El Escorial. Los autores de catálogos provinciales recientes (Morales, 2003; López Jiménez, 2007) se limitan a recoger su presencia sin indicar localidades precisas, aunque la región está recogida en Flora iberica (Gonçalves, 1990: 95) apoyada por material de herbario reciente. 
Las referencias en el entorno de la región de Madrid corresponden a la provincia de Ávila, en el área del embalse del Burguillo (Rivas Goday, 1971; Molina Moreno, 1992); en la de Segovia, en diversas localidades de la zona central de la provincia (Romero \& Rico, 1986; 1989; García Adá, 1995); en la de Guadalajara, en los arenales silíceos de La Fuensaviñán (Monge, 1985); en la de Cuenca, en localidades de la Serranía (García Cardo, 2011) y en la de Toledo, en Polán (Pomata, 1882).

El material depositado en MA cubre un periodo desde c. 1800 hasta casi la actualidad, con un lapso importante durante buena parte del siglo XIX y principios del XX: Madrid, s.f., [A.J. Cavanilles s.n.] (MA-30198); Prope Matritum ín sabulosis. Escorial, s.f. [J.D. Rodríguez s.n. (ver Carrasco \& al., 2000)] (MA-720474, ex Real Colegio del Alfonso II de San Lorenzo de El Escorial); Ex Matriti suburbiis, 1801, L. Neé s.n. (MA-30197). Más recientemente: Madrid, orillas del Manzanares, VII-1929, A. Aterido s.n. (MA-162065); Madrid: Quijorna, eriales y barbechos, 15-VII-1971, J. Izco s.n. (MA-217576); Madrid, Parque del Oeste, Rosaleda, ruderal en suelo arenoso, 27-VI-1981, S. Castroviejo SC5819 (MA-226618); Madrid, Parque del Oeste, Rosaleda, suelos arenosos, s.f., E. Valdés-Bermejo s.n. (MA435236).

Entre julio y agosto de 2017, en unas visitas de prospección por los aledaños del río Guadalix a su paso por la localidad de San Agustín encontramos dos poblaciones de este taxon. La primera, situada en la margen izquierda del río, en un campo de cultivo de cereal ya cosechado, sobre terrenos arenoso propio de una terraza fluvial, en compañía de Polycnemum arvense L. La segunda, en la otra margen, se encuentra sobre suelos arenosos y muy lavados, en la zona cerrada al tráfico de un área recreativa, en compañía de Tribulus terrestris L. Ambas situaciones se ajustan con lo indicado por Gonçalves (1990) y García Cardo (2011) sobre su preferencia por los suelos arenosos de inundación estacional, que además pueden haber sido cultivados (Belmonte, 1983).

\section{Agradecimientos}

A Esther García Guillén, por facilitarnos el acceso al Archivo de RJB en busca de los manuscritos de Palau y a Mauricio Velayos por su ayuda con las caligrafías de A.J. Cavanilles y J.D. Rodríguez.

\section{Referencias}

ANTHOS (2017). Sistema de información de las plantas de España. Real Jardín Botánico, CSIC- Fundación
Biodiversidad. Recurso electrónico en www.anthos. es. Consulta realizada en agosto de 2017.

Asso, I. (1801). Continuacion de las observaciones de Historia Natural hechas en España y en América por Pedro Loefling: Traducidas por D. Ignacio de Asso. Anales de Ciencias Naturales, 4(11), 155-191.

Belmonte, D. (1983). Datos florísticos sobre la comarca de las Corchuelas (Parque Natural de Monfragüe, Cáceres, España), III. Lazaroa, 5, 315-317.

Carrasco, M.A., Santamaría, C., Pérez Martín, R. \& Perea, D. (2000). Cistaceae de José Demetrio Rodríguez (1780?-1846) en el Herbario del Real Colegio Alfonso XII de San Lorenzo de El Escorial. Botanica Complutensis, 24, 81-88.

Cavanilles, J.A. (1803a). Elenchus Plantarum Horti Regii Botanici Matritensis. Anno M.DCCC.III. Madrid, Real Jardín Botánico.

Cavanilles, J.A. (1803b). Géneros y especies de plantas demostradas en las lecciones públicas del año 1802. Madrid, Imprenta Real.

Colmeiro, M. (1849). Apuntes para la flora de las dos Castillas. Madrid, Librería Angel Calleja.

Colmeiro, M. (1886). Enumeración y revisión de las plantas de la Península Hispano-Lusitana é islas Baleares [...] Tomo II. Madrid, Imprenta de la viuda é hija de Fuentenebro.

Cutanda, V. \& Amo, M. (1848). Manual de botánica descriptiva. Madrid, Imprenta de D.S. Saunaque.

Cutanda, V. (1861). Flora compendiada de Madrid y su provincia, o descripción sucinta de las plantas vasculares que espontáneamente crecen en este territorio. Madrid, Imprenta Nacional.

Gandoger, M. (1896). Sur la découverte de plusieurs genres et espèces nouvelles pour la flore espagnole. Bulletin de la Société Botanique de France, 43, 681692.

García Adá, R. (1995). Estudio de la flora y vegetación de las cuencas alta y media de los ríos Eresma, Pirón y Cega (Segovia). Madrid, Tesis doctoral, Fac. Biología, Univ. Complutense.

García Cardo, Ó. (2011). Aportaciones a la flora de la provincia de Cuenca, IV. Flora Montiberica 48, 52-64.

Gómez Ortega, C. (1772). Indice de las plantas que se han sembrado en el Real Jardin Botánico en este año de 1772. Madrid, Real Jardín Botánico.

Gómez Ortega, C. (1796). Elenchus Plantarum Horti Regii Botanici Matritensis. Anno M.DCC.XCVI. Madrid, Real Jardín Botánico.

Gonçalves, M.L. (1990). Mollugo L. In S. Castroviejo et al. (Eds), Flora iberica 2 (pp. 94-95). Madrid, Real Jardín Botánico.

Lagasca, M. (1816). Elenchus plantarum, quae in Horto Regio Botanico Matritensi colebantur anno MDCCCXV. Madrid, Imprenta Real.

Laínz, M. (1981). In Florae Europaeae Atlantem chorologicae animadversiones, II. Anuário da Sociedade Broteriana, 47, 67-75.

Löfling, P. (1758). Iter Hispanicum. Stockholm, Ttryckt på Direct. Lars Salvii Kostnad.

López Jiménez, N. (2007). Las plantas vasculares de la Comunidad de Madrid. Catálogo florístico, claves dicotómicas y estudio detallado de la familia 
Compositae Giseke. Tesis doctoral, Fac. de Biología, Univ. Complutense de Madrid, Madrid.

Medina, L., Barberá, P., Buira, A., Tomé, F.J. \& Aedo, C. (2015). Limosella aquatica L. en la Comunidad de Madrid. Acta Botanica Malacitana, 40, 211-257.

Minuart, J. (1739). Cerviana sub auspiciis illustrissimi viri D.D. Josephi Cervi. Monograph., 2 pp.

Molina Moreno, J.R. (1992). Flora y vegetación del valle de Iruelas (Ávila). Cuadernos Abulenses, 18, 11-149.

Monge, C. (1985). Fragmenta chorologica occidentalia, 73-86. Anales del Jardín Botánico de Madrid, 42, 239240.

Morales, R. (2003). Catálogo de plantas vasculares de la Comunidad de Madrid. Botanica Complutensis, 27, 31-70.

Pérez, F. (1930). Antófitas de San Lorenzo de El Escorial. El Escorial, Imprenta del Real Monasterio.

Pomata, E. (1882): Catálogo de plantas recolectadas al estado espontáneo en la provincia de Toledo. Anales de la Sociedad Española de Historia Natural, 11(2-3), 241-306.

Reuter, G.F. (1843). Essai sur la végétation de la Nouvelle
Castille. Geneve, Imprimerie de Jules-Gme Fick.

Rivas Goday, S. (1971). Revisión de las comunidades hispanas de la clase Isoeto-Nanojuncetea Br.-BI. \& Tx. 1943, Anales del Instituto Botánico A.J. Cavanilles, 27, 225-276.

Romero, T. \& Rico, E. (1986). Algunas plantas de los arenales de la cuenca del Duero. Lazaroa, 9, 159-165.

Romero, T. \& Rico, E. (1989). Flora de la cuenca del río Duratón. Ruizia, 8, 7-438.

Salgueiro González, F.J. (1998). Estudio sobre los herbarios históricos de la Universidad de Sevilla. Tesis doctoral, Fac. de Biología, Univ. de Sevilla, Sevilla.

USDA-NRCS (2017). The PLANTS Database (http:// plants.usda.gov, consultada en agosto 2017). National Plant Data Team, Greensboro, NC 27401-4901 USA.

Vincent, M.A. (2003). Molluginaceae Rafinesque. In Flora of North America Editorial Committee (Eds.), Flora of North America North of Mexico 4 (pp. 509512). New York and Oxford.

Willkomm, H.M. \& Lange, J.M.C. (1874-1880). Prodromus florae Hispanicae [...] Volumen III. Stuttgartiae: Sumtibus E. Schweizerbart. 